KIAS-P11032

\title{
Killing Spinors for the Bosonic String
}

\author{
H. Lü ${ }^{1,2}$ and Zhao-Long Wang ${ }^{3}$ \\ ${ }^{1}$ China Economics and Management Academy \\ Central University of Finance and Economics, Beijing 100081, China \\ ${ }^{2}$ Institute for Advanced Study, Shenzhen University \\ Nanhai Ave 3688, Shenzhen 518060, China \\ ${ }^{3}$ School of Physics, Korea Institute for Advanced Study, Seoul 130-722, Korea
}

\begin{abstract}
$\underline{\text { ABSTRACT }}$
We obtain the effective action for the bosonic string with arbitrary Yang-Mills fields, up to the $\alpha^{\prime}$ order, in general dimensions. The form of the action is determined by the requirement that the action admit well-defined Killing spinor equations, whose projected integrability conditions give rise to the full set of equations of motion. The success of the construction suggests that the hidden "pseudo-supersymmetry" associated with the Killing spinor equations may be a property of the bosonic string itself.
\end{abstract}


One of the most important advances in quantum gravity was the invention of string theory. Quantum consistency in general requires that string theories be supersymmetric in the critical dimension ten 1 With the discovery of the AdS/CFT correspondence [2, the application of string theory goes beyond quantum gravity, but also provides new understandings and techniques in non-perturbative quantum field theories including nuclear and condensed matter physics. However, these low-energy arenas are necessarily non-supersymmetric, and hence the hitherto abandoned bosonic string theory may provide an alternative and useful approach.

One advantage of supersymmetry is that it provides a powerful organizing tool for the non-perturbative region. In particular the characteristic properties of the BPS solutions of supergravities, such as the mass-charge relation, are expected to survive the higherorder quantum corrections. The defining property for the BPS solutions is that they admit Killing spinors. Killing spinors, however, were introduced in ordinary Riemannian geometry which predates supersymmetry. Killing spinors in supergravities are generalizations of those in the Riemannian geometry. They all have an important defining property that certain projected integrability conditions for the Killing spinor equations give rise to the full set of equations of motion of the bosonic fields. This property is a necessary condition for constructing supergravities, and conversely, all supergravities have consistent Killing spinor equations. It is natural to ask whether there exist non-supersymmetric theories other than pure gravities that also admit well-defined Killing spinors.

Recently, an intriguing connection between the consistency of the Kluza-Klein sphere reduction and Killing spinors was observed in [3]. Inspired by this and also the fact that it is consistent to perform $S^{3}$ and $S^{D-3}$ reductions on the effective action of the bosonic string [4], Killing spinor equations were proposed for this action [3]. It was shown that the projected integrability condition gives rise precisely to the full set of equations of motion. For non-supersymmetric theories, such examples are uncommon [5]. The only known nontrivial examples are pure gravity, certain scalar-gravity theories [6] and the bosonic string. This implies that the bosonic string has a non-trivial generalized geometric structure arising from the "pseudo-supersymmetry", a terminology we introduce here to refer to the hidden symmetry associated with the existence of well-defined Killing spinor equations in nonsupersymmetric theories. From the AdS/CFT point of view, where the bulk gravity is classical, the pseudo-supersymmetric effective action of the bosonic string can be put on an

\footnotetext{
${ }^{1}$ Exotic tachyon-free string theory without supersymmetry is also possible with appropriate discrete torsion introduced in the partition function [1.
} 
equal footing as in supergravity.

The pseudo-supersymmetry obtained in [3] is for the tree-level action of the bosonic string. If the pseudo-supersymmetry is indeed a generic stringy property, we would expect that the Killing spinor equations should remain well-defined when higher-order corrections are included. In this paper, we extend the discussion of [3] by considering the $\alpha^{\prime}$ correction to the low-energy effective action. We start by first introducing arbitrary Yang-Mills fields, which originate in diverse ways in string theory. For an example, wrapping the string on singular cycles of the internal manifold can lead to gauge symmetry enhancement. One can also consider hybrid mixing between the left- and right-moving sectors, with the mismatching dimensions compactified on some suitable self-dual lattices, à la heterotic string [7. The effective action with arbitrary Yang-Mills fields in $D$ dimensions is given by

$$
\mathcal{L}_{D}=\sqrt{-g}\left(R-\frac{1}{2}(\partial \phi)^{2}-\frac{1}{12} e^{a \phi} G_{(3)}^{2}-\frac{1}{4} e^{\frac{1}{2} a \phi} \operatorname{tr}^{\prime} F_{(2)}^{2}\right)
$$

where $a^{2}=8 /(D-2)$ and the various form fields are given by

$$
\begin{aligned}
G_{(3)} & =d B_{(2)}-\frac{1}{2} \omega_{(3)}, \quad \omega_{(3)}=\operatorname{tr}^{\prime}\left(F_{(2)} \wedge A_{(1)}-\frac{1}{3} A_{(1)} \wedge A_{(1)} \wedge A_{(1)}\right), \\
F_{(2)} & =d A_{(1)}+A_{(1)} \wedge A_{(1)}, \quad d G_{(3)}=-\frac{1}{2} \operatorname{tr}^{\prime}\left(F_{(2)} \wedge F_{(2)}\right) .
\end{aligned}
$$

The Yang-Mills 1-forms are defined by $A_{(1)}=A^{I} T_{I}$, where the generators $T_{I}$ are antihermitian, obeying the Lie algebra $\left[T_{I}, T_{J}\right]=f^{K}{ }_{I J} T_{K}$, and normalized in the fundamental representation as $\operatorname{tr}\left(T_{I} T_{J}\right) \equiv \beta \delta_{I J}$. Then the trace $\operatorname{tr}^{\prime}$ is defined by $\operatorname{tr}^{\prime}=\frac{1}{\beta} \operatorname{tr}$. Note that for simplicity, we have set the Yang-Mills coupling to unity.

We propose that the defining equations for the Killing spinors for (11) are given by

$$
\begin{aligned}
D_{M} \epsilon+\frac{1}{96} e^{\frac{1}{2} a \phi}\left(a^{2} \Gamma_{M} \Gamma^{N P Q}-12 \delta_{M}^{N} \Gamma^{P Q}\right) G_{N P Q} \eta & =0, \\
\Gamma^{M} \partial_{M} \phi \eta+\frac{1}{12} a e^{\frac{1}{2} a \phi} \Gamma^{M N P} G_{M N P} \eta & =0, \\
\Gamma^{M_{1} M_{2}} F_{M_{1} M_{2}} \eta & =0 .
\end{aligned}
$$

When $D=10$, these are precisely the Killing spinor equations for $D=10, \mathcal{N}=1$ supergravity [8] with Yang-Mills matter multiplets. Let us now examine the consistency of these equations. For Riemannian Killing spinors, satisfying $D_{M} \epsilon=0$, the integrability condition is given by $\left[D_{M}, D_{N}\right] \epsilon=\frac{1}{4} R_{M N P Q} \Gamma^{P Q} \epsilon=0$. The projected integrability condition, namely $\Gamma^{M}\left[D_{M}, D_{N}\right] \epsilon=\frac{1}{2} R_{M N} \Gamma^{M} \epsilon=0$, is satisfied by virtue of the Einstein equation. The projected integrability conditions for the equations (3)-(5) are much more involved. We find that the condition associated with $\Gamma^{M}\left[D_{M}, D_{N}\right] \eta$ is given by

$$
\left[R_{M N}-\frac{1}{2} \partial_{M} \phi \partial_{N} \phi-\frac{1}{4} e^{a \phi}\left(G_{M N}^{2}-\frac{2}{3(D-2)} G^{2} g_{M N}\right)\right.
$$




$$
\begin{gathered}
\left.-\frac{1}{2} e^{\frac{1}{2} a \phi} \operatorname{tr}^{\prime}\left(F_{M N}^{2}-\frac{1}{2(D-2)} F^{2} g_{M N}\right)\right] \Gamma^{N} \eta \\
-\frac{e^{\frac{1}{2} a \phi}}{6(D-2)}\left(\nabla_{N} G_{M_{1} M_{2} M_{3}}+\frac{3}{4} F_{N M_{1}} F_{M_{2} M_{3}}\right)\left(\Gamma_{M} \Gamma^{N M_{1} M_{2} M_{3}}-2(D-2) \delta_{M}^{[N} \Gamma^{\left.M_{1} M_{2} M_{3}\right]}\right) \eta \\
-\frac{e^{-\frac{1}{2} a \phi}}{2(D-2)} \nabla_{N}\left(e^{a \phi} G^{N}{ }_{M_{2} M_{3}}\right)\left(\Gamma_{M} \Gamma^{M_{2} M_{3}}-(D-2) \delta_{M}^{M_{2}} \Gamma^{M_{3}}\right) \eta=0 .
\end{gathered}
$$

Acting with $\Gamma^{N} D_{N}$ on (4) and (5) gives rise to

$$
\begin{aligned}
& \left(\nabla^{2} \phi-\frac{1}{12} a e^{a \phi} G^{2}-\frac{1}{8} a e^{\frac{1}{2} a \phi} F^{2}\right) \eta+\frac{1}{12} a e^{\frac{1}{2} a \phi} \Gamma^{N M_{1} M_{2} M_{3}}\left(\nabla_{N} G_{M_{1} M_{2} M_{3}}\right. \\
& \left.+\frac{3}{4} F_{N M_{1}} F_{M_{2} M_{3}}\right) \eta+\frac{1}{4} a e^{-\frac{1}{2} a \phi} \Gamma^{M_{2} M_{3}} \nabla_{N}\left(e^{a \phi} G^{N} M_{2} M_{3}\right) \eta=0,
\end{aligned}
$$

and

$$
\Gamma^{M M_{1} M_{2}} \hat{D}_{M} F_{M_{1} M_{2}} \eta+\left[2 e^{-\frac{1}{2} a \phi} \hat{D}^{M}\left(e^{\frac{1}{2} a \phi} F_{M N}\right) \eta+F^{M_{1} M_{2}} G_{M_{1} M_{2} N}\right] \Gamma^{N} \eta=0 .
$$

Here, $\hat{D} \equiv d+[A$,$] . In the above derivation, we have used the important identity$

$$
\Gamma_{N M_{1}}^{M_{2} M_{3}} F^{N M_{1}} F_{M_{2} M_{3}} \eta=\left(\frac{1}{2}\left\{\Gamma_{N M_{1}}, \Gamma^{M_{2} M_{3}}\right\}+2 \delta_{[N}^{M_{2}} \delta_{\left.M_{1}\right]}^{M_{3}}\right) F^{N M_{1}} F_{M_{2} M_{3}} \eta=2 F^{2} \eta,
$$

which enables us to turn the $F_{(2)} \wedge F_{(2)}$ structure from the Bianchi identity for $G_{(3)}$ into the $F_{(2)}^{2}$ term in the Einstein equations of motion. Analogously, we have used identity $\Gamma_{M_{1}}{ }^{M_{2} M_{3}} F_{M_{2} M_{3}} \eta=-2 \Gamma^{M_{2}} F_{M_{1} M_{2}} \eta$. Thus the full set of equations of motion emerges from the projected integrability conditions together with the Bianchi identity for the 3-form. This demonstrates that the concept of the Killing spinors is well defined in the effective theory, even with the Yang-Mills fields. The results without the Yang-Mills were previously obtained in [3].

As in the construction of extended supergravities, there is a similarity between the curvature 2-form and the Yang-Mills fields, such that the supersymmetrization of curvaturesquare term follows by straightforward analogy with that for the Yang-Mills [9]. To proceed, it is advantageous to work with the theory in the string frame, which is defined by

$$
d s_{\text {string }}^{2}=e^{-\frac{1}{2} a \phi} d s_{\text {Einstein }}^{2}
$$

If we now define $\Phi=-\phi / a$, the Lagrangian becomes

$$
\mathcal{L}=\sqrt{-g} e^{-2 \Phi}\left(R+4(\partial \Phi)^{2}-\frac{1}{12} G_{(3)}^{2}-\frac{1}{4} \operatorname{tr}^{\prime} F_{(2)}^{2}\right)
$$

The defining equations for the Killing spinors, which are scaled by a factor $e^{-\frac{1}{8} a \phi}$ in the string frame, are now given by

$$
D_{M}\left(\omega_{-}\right) \eta=0, \quad \Gamma^{M} \partial_{M} \Phi \eta-\frac{1}{12} \Gamma^{M N P} G_{M N P} \eta=0, \quad \Gamma^{M_{1} M_{2}} F_{M_{1} M_{2}} \eta=0,
$$


where $\omega_{-}$is the torsional spin connection, defined as

$$
\omega_{\mu \pm}^{a b}=\omega_{\mu}^{a b} \pm \frac{1}{2} G_{\mu}^{a b} .
$$

A major advantage of the string frame is that there is no manifest dimensional dependence in either the action or the Killing spinor equations. At the $\alpha^{\prime}$ order, anomaly cancelation requires that the quadratic curvature terms enter the Bianchi identity of the 3 -form in the form of $d G_{(3)} \sim \frac{1}{2} \alpha\left(\operatorname{tr}\left(R_{(2)} \wedge R_{(2)}\right)-\operatorname{tr}^{\prime}\left(F_{(2)} \wedge F_{(2)}\right)\right)$, where $R_{(2)}=d \omega+\omega \wedge \omega$ is the curvature 2-form and $\operatorname{tr}\left(R_{(2)} \wedge R_{(2)}\right) \equiv\left(R_{(2)}\right)^{a}{ }_{b}\left(R_{(2)}\right)^{b} a$. (Note that for the ten-dimensional superstring, $\alpha=\frac{1}{2} \alpha^{\prime}$, and we expect this to hold in general.) This suggests that we do not need to modify the Killing spinors equations (12), but instead add an additional projection associated with the curvature 2-form $\grave{a}$ la the Yang-Mills. This projection in fact already exists at the $\alpha^{\prime}$ order, since [10]

$$
0=\left[D_{M}\left(\omega_{-}\right), D_{N}\left(\omega_{-}\right)\right] \eta=\frac{1}{4} R_{M N}^{a b}\left(\omega_{-}\right) \Gamma_{a b} \eta=\frac{1}{4} R_{M N}^{a b}\left(\omega_{+}\right) \Gamma_{a b} \eta+\mathcal{O}(\alpha) .
$$

This projection is analogous to that for the Yang-Mills fields (5), and hence the proper Bianchi identity for the 3-form is given by

$$
d G_{(3)}=\frac{1}{2} \alpha\left(\operatorname{tr}\left(R_{(2)}\left(\omega_{+}\right) \wedge R_{(2)}\left(\omega_{+}\right)\right)-\operatorname{tr}^{\prime}\left(F_{(2)} \wedge F_{(2)}\right)\right) .
$$

Note that we adopt the supergravity convention that the torsionful Riemann tensors are defined as $\left(R_{(2)}\left(\omega_{+}\right)\right)^{a}{ }_{b}=\frac{1}{2} R_{M N}{ }^{a}{ }_{b}\left(\omega_{+}\right) d x^{M} \wedge d x^{N}$. Following the same strategy as in the earlier calculation, we find that the full set of equations of motion can now be obtained by the projected integrability conditions, together with the Bianchi identity (15). They are given by

$$
\begin{aligned}
& R-4(\partial \Phi)^{2}+4 \square \Phi-\frac{1}{12} G_{(3)}^{2}-\frac{1}{4} \alpha\left(\operatorname{tr}^{\prime} F_{(2)}^{2}-R_{M N A B}\left(\omega_{+}\right) R^{M N A B}\left(\omega_{+}\right)\right)=0, \\
& R_{M N}+2 \nabla_{M} \nabla_{N} \Phi-\frac{1}{4} G_{M N}^{2}-\frac{1}{2} \alpha\left(\operatorname{tr}^{\prime} F_{M N}^{2}-R_{M P A B}\left(\omega_{+}\right) R_{N} P A B\left(\omega_{+}\right)\right)=0, \\
& d\left(e^{-2 \Phi} * G_{(3)}\right)=0, \quad \hat{D}\left(e^{-2 \Phi} * F_{(2)}\right)+(-1)^{D} e^{-2 \Phi} F_{(2)} \wedge * G_{(3)}=0 .
\end{aligned}
$$

Up to the $\alpha^{\prime}$ order, these equations can be derived from the Lagrangian

$$
\mathcal{L}_{D}=\sqrt{-g} e^{-2 \Phi}\left[R+4(\partial \phi)^{2}-\frac{1}{12} G_{(3)}^{2}-\frac{1}{4} \alpha\left(\operatorname{tr}^{\prime} F_{(2)}^{2}-R_{M N A B}\left(\omega_{+}\right) R^{M N A B}\left(\omega_{+}\right)\right)\right] .
$$

This Lagrangian in general dimensions takes the exact form of the bosonic effective action of the heterotic string with curvature-squard terms [10]. The equations of motion (16) also take the same form as those in the corresponding supergravity [11. Note that the terms arising from the variation of $\omega_{+}$in the torsional Riemann tensor do not appear in the 
equations of motion (16). These terms could at least contribute to the $\alpha^{\prime}$ order. As in the $R^{2}$ supergravity in $D=10$ [10], at the $\alpha^{\prime}$ order, we find that they vanish by virtue of the leading-order equations of motion. Thus the effective action of the bosonic string are now fixed, up to the $\alpha^{\prime}$ order, by the assumption that the Killing spinors can be well defined. Conversely the above derivation also indicates strongly that the pseudo-supersymmetry may be a property of the full bosonic string.

We now turn to the solutions of the bosonic string. Let us first set $\alpha^{\prime}=0$. The theory admits the electric string solution

$$
\begin{aligned}
d s_{\mathrm{str}}^{2} & =H^{-1}\left(-d t^{2}+d x^{2}\right)+d r^{2}+r^{2} d \Omega_{D-3}^{2}, \\
e^{2 \Phi} & =H^{-1}, \quad F_{(3)}=d t \wedge d x \wedge d H^{-1}, \quad H=1+\frac{Q}{r^{D-4}}
\end{aligned}
$$

and the magnetic $(D-5)$-brane

$$
\begin{aligned}
d s_{\mathrm{str}}^{2} & =\eta_{\mu \nu} d x^{\mu} d x^{\nu}+H\left(d r^{2}+r^{2} d \Omega_{3}^{2}\right) \\
e^{2 \Phi} & =H, \quad e^{-2 \Phi} * F_{(3)}=d H^{-1} \wedge d^{(D-4)} x, \quad H=1+\frac{P}{r^{2}}
\end{aligned}
$$

and their intersection

$$
\begin{aligned}
d s_{\mathrm{str}}^{2} & =H_{1}^{-1}\left(-d t^{2}+d x^{2}\right)+H_{2}\left(d r^{2}+r^{2} d \Omega_{3}^{2}\right)+d x^{i} d x^{i}, \\
e^{2 \Phi} & =\frac{H_{2}}{H_{1}}, \quad F_{(3)}=d t \wedge d x \wedge d H^{-1}+e^{2 \Phi} * d t \wedge d x \wedge d^{(D-6)} x \wedge d H_{2}^{-1}, \\
H_{1} & =1+\frac{Q}{r^{2}}, \quad H_{2}=1+\frac{P}{r^{2}} .
\end{aligned}
$$

In the decoupling limit when the "1" in the harmonic functions $H_{i}$ can be dropped, the metric (20) becomes $\mathrm{AdS}_{3} \times S^{3} \times \mathbb{R}^{D-6}$. As in supergravities, we shall call these solutions "BPS" since they preserve fractions of the maximally-allowed Killing spinors in the vacuum.

The Bianchi identity for $G_{(3)}$ implies that the $(D-5)$-brane can be supported by YangMills instantons. For a single $S U(2)$ instanton, the Yang-Mills fields are given by $A_{(1)}^{i}=$ $a^{2} /\left(r^{2}+a^{2}\right) \sigma_{i}$, where $\sigma_{i}$ are the $S U(2)$ left-invariant 1-forms. The $S^{3}$ metric in (19) can be expressed as $d \Omega_{3}^{2}=\frac{1}{4}\left(\sigma_{1}^{2}+\sigma_{2}^{2}+\sigma_{3}^{2}\right)$. The $(D-5)$-brane takes the same form as (19)) but with $H$ now given by

$$
H=1+\frac{r^{2}+2 a^{2}}{\left(r^{2}+a^{2}\right)^{2}} .
$$

This solution in the heterotic string was obtained in [12]. For multi-instanton supported solutions, see [13].

We now examine the $\alpha^{\prime}$ corrected solutions. It turns out that, up to the $\alpha^{\prime}$ order, (19) is unmodified as a solution for the Lagrangian (17). This is because the torsion for this solution is parallelizing and hence the curvature 2 -form $R_{(2)}\left(\omega_{+}\right)$vanishes. If the existence 
of the Killing spinors would restrict the higher-order corrections to be scalar polynomials of $R_{(2)}\left(\omega_{+}\right)$, the solution (19) would survive all the quantum corrections. This was first observed for heterotic string in [12], and we expect the same may be true for the bosonic string with Killing spinors.

In supergravities, the supersymmetry implies the existence of the superspace geometry. The solutions can be classified by a generalized holonomy. The generalized holonomy for the M-theory was studied in [14, 15]. The existence of the well-defined Killing spinors for the bosonic string suggests that the theory may also have a generalized geometrical structure which can be called pseudo-superspace. The Killing spinor equations in (12) imply that the generalized holonomy remains in the same $S O(1, D-1)$ as Einstein gravity since it merely adds a totally-antisymmetric torsion to the usual spin connection. We now examine the reduced holonomy for the $\frac{1}{2}$-BPS solutions. We first look at the electric string solution. We can replace the metric of the $(D-2)$-dimensional transverse space by the one in the Cartesian coordinates $d y^{m} d y^{m}$, in which $H$ is an arbitrary harmonic function. For this background, the "super"-covariant derivative is given by

$$
\mathcal{D}_{\mu}=\partial_{\mu}-\frac{1}{2} H^{-\frac{3}{2}} \partial_{m} H P^{+} \Gamma_{\hat{\mu}}^{\hat{m}}, \quad \mathcal{D}_{m}=\partial_{m}-\frac{1}{4} H \partial_{m} H^{-1} \Gamma^{\hat{0} \hat{1}},
$$

where the projection operator is given by $P^{ \pm}=\frac{1}{2}\left(1 \pm \Gamma^{\hat{0} \hat{1}}\right)$. Letting $\mathcal{M}_{M N}=\left[\mathcal{D}_{M}, \mathcal{D}_{N}\right]$, we find

$$
\mathcal{M}_{\mu \nu}=0=\mathcal{M}_{m n}, \quad \mathcal{M}_{\mu m}=\frac{1}{2} H^{-\frac{1}{2}} \partial_{n} \partial_{m} \ln H P^{+} \Gamma_{\hat{\mu}}^{\hat{n}} .
$$

Since only the commuting generators $K_{\hat{\mu}}^{\hat{m}}=P^{+} \Gamma_{\hat{\mu}}^{\hat{m}}$ are present, the reduced holonomy for the electric string solution is given by

$$
\mathcal{H}_{\text {string }}=\mathbb{R}^{D-2} \text {. }
$$

The analysis for the magnetic $(D-5)$-brane is similar. After replacing the transverse space in (19) with the Cartesian system, the "super"-covariant derivative is given by

$$
\mathcal{D}_{\mu}=\partial_{\mu}, \quad \mathcal{D}_{m}=\partial_{m}+\frac{1}{2} H^{-1} \partial_{n} H P^{+} \Gamma_{\hat{m}}^{\hat{n}},
$$

where the projection operator is given by $P^{ \pm}=\frac{1}{2}\left(1 \pm \Gamma^{\hat{1} \hat{2} \hat{3} \hat{4}}\right)$. We now have

$$
\begin{aligned}
\mathcal{M}_{\mu \nu}= & 0, \quad \mathcal{M}_{\mu m}=0, \\
\mathcal{M}_{m n}= & \frac{1}{2}\left(\partial_{m} \partial_{n_{1}} f-\left(\partial_{m} f\right)\left(\partial_{n_{1}} f\right)\right) P^{+} \Gamma_{\hat{n}}^{\hat{n}_{1}}-\frac{1}{2}\left(\partial_{n} \partial_{n_{1}} f-\left(\partial_{n} f\right)\left(\partial_{n_{1}} f\right)\right) P^{+} \Gamma_{\hat{m}}^{\hat{n}_{1}} \\
& -\frac{1}{2}\left(\partial^{n_{1}} f\right)\left(\partial_{n_{1}} f\right) P^{+} \Gamma_{\hat{m} \hat{n}},
\end{aligned}
$$

where $f=\ln H$. The non-vanishing generators are $T_{\hat{m} \hat{n}}=P^{+} \Gamma_{\hat{m} \hat{n}}$. Since

$$
\left[P^{+} \Gamma_{\hat{m}_{1} m_{2}}, P^{+} \Gamma_{\hat{n}_{1} \hat{n}_{2}}\right]=P^{+}\left[\Gamma_{\hat{m}_{1} m_{2}}, \Gamma_{\hat{n}_{1} \hat{n}_{2}}\right]
$$


it follows that $T_{\hat{m} \hat{n}}$ generates the $s o(4)$ algebra. Thus the reduced holonomy for the $(D-5)$ brane is given by

$$
\mathcal{H}_{(D-5) \text {-brane }}=S O(4)_{+}
$$

where + refers to the sign of the $P_{+}$projection. For the $\frac{1}{4}$-BPS intersecting solution, we find that the reduced holonomy is $\mathcal{H}=S O(4)_{+} \ltimes 2 \mathbb{R}^{\left(2_{s}\right)}$, where $\mathbb{R}^{\left(2_{s}\right)}$ is the two-dimensional spinor representation of the $S O(4)_{+}$.

To conclude, we obtain the effective action for the bosonic string up to the $\alpha^{\prime}$ order. The guiding principle in the construction is that the theory has well-defined Killing spinor equations, whose projected integrability condition yields the full set of equations of motion. The success of our construction suggests that the hidden pseudo-supersymmetry associated with the Killing spinor equations may be a property of the full bosonic string. The pseudo-supersymmetry enables us to classify solutions with respect to the fractions of the surviving Killing spinors. These solutions are characterized by the different reduced holonomy groups which are subgroups of $S O(1, D-1)$. It is tempting to conjecture that these hidden symmetry may cure some of the pathologies in the bosonic string. Furthermore, as in the case of the superstring and M-theory, the classical solutions with Killing spinors may also be protected from quantum corrections and hence provide tools for studying the non-perturbative aspects of the bosonic string.

\section{Acknowledgement}

We are grateful to Haishan Liu, Yi Pang and Chris Pope for useful discussions.

\section{References}

[1] J. Polchinski, String theory, Cambridge University Press (1998).

[2] J.M. Maldacena, The Large $N$ limit of superconformal field theories and supergravity, Adv. Theor. Math. Phys. 2, 231 (1998) [Int. J. Theor. Phys. 38, 1113 (1999)] arXiv:hep-th/9711200.

[3] H. Lü, C.N. Pope and Z.L. Wang, Pseudo-supersymmetry, consistent sphere reduction and Killing spinors for the bosonic string, arXiv:1105.6114 [hep-th].

[4] M. Cvetič, H. Lü and C.N. Pope, Consistent Kaluza-Klein sphere reductions, Phys. Rev. D 62, 064028 (2000) arXiv:hep-th/0003286. 
[5] H. Lü and Z.L. Wang, Pseudo-Killing Spinors, Pseudo-supersymmetric p-branes, Bubbling and Less-bubbling AdS Spaces, arXiv:1103.0563 [hep-th].

[6] D.Z. Freedman, C. Nunez, M. Schnabl and K. Skenderis, Fake supergravity and domain wall stability, Phys. Rev. D69, 104027 (2004), hep-th/0312055.

[7] D.J. Gross, J.A. Harvey, E.J. Martinec and R. Rohm, The heterotic string, Phys. Rev. Lett. 54, 502 (1985).

[8] E. Bergshoeff, M. de Roo, B. de Wit and P. van Nieuwenhuizen, Ten-dimensional Maxwell-Einstein supergravity, its currents, and the issue of its auxiliary fields, Nucl. Phys. B195, 97 (1982).

[9] E. Bergshoeff, A. Salam and E. Sezgin, A Supersymmetric $R^{2}$ action in six-dimensions and torsion, Phys. Lett. B 173, 73 (1986).

[10] E.A. Bergshoeff and M. de Roo, The quartic effective of the heterotic string and supersymmetry, Nucl. Phys. B 328, 439 (1989).

[11] K. Becker and S. Sethi, Torsional heterotic geometries, Nucl. Phys. B 820, 1 (2009) arXiv:0903.3769 [hep-th]].

[12] A. Strominger, Heterotic solitons, Nucl. Phys. B 343, 167 (1990) [Erratum-ibid. B 353, 565 (1991)].

[13] E. Lima, H. Lü, B.A. Ovrut and C.N. Pope, Instanton moduli and brane creation, Nucl. Phys. B 569, 247 (2000) arXiv:hep-th/9903001.

[14] M.J. Duff and J.T. Liu, Hidden space-time symmetries and generalized holonomy in M theory, Nucl. Phys. B 674, 217 (2003) arXiv:hep-th/0303140].

[15] A. Batrachenko, M.J. Duff, J.T. Liu and W.Y. Wen, Generalized holonomy of M theory vacua, Nucl. Phys. B 726, 275 (2005) arXiv:hep-th/0312165]. 